Check for updates

Cite this: Phys. Chem. Chem. Phys. 2020, 22, 23215

Received 14th July 2020 ,

Accepted 22nd September 2020

DOI: 10.1039/d0cp03750h

rsc.li/pccp

\title{
Thermochemical unification of molecular descriptors to predict radical hydrogen abstraction with low computational cost $\dagger$
}

\author{
Tom M. Nolte, (D) *ab Thomas Nauser, (D) ${ }^{a}$ Lorenz Gubler, (D) ${ }^{c}$ A. Jan Hendriks ${ }^{b}$ and \\ Willie J. G. M. Peijnenburg (iD) de
}

\begin{abstract}
Chemistry describes transformation of matter with reaction equations and corresponding rate constants. However, accurate rate constants are not always easy to get. Here we focus on radical oxidation reactions. Analysis of over 500 published rate constants of hydroxyl radicals led us to hypothesize that a modified linear free-energy relationship (LFER) could be used to predict rate constants speedily, reliably and accurately. LFERs correlate the Gibbs activation-energy with the Gibbs energy of reaction. We calculated the latter as the sum of one-electron transfer and, if appropriate, proton transfer. We parametrized specific transition state effects to orbital delocalizability and the polarity of the reactant. The calculation time for 500 reactions is less than 8 hours on a standard desktop-PC. Rate constants were also calculated for hydrogen and methyl radicals; these controls show that the predictions are applicable to a broader set of oxidizing radicals. An accuracy of $30-40 \%$ (standard deviation) with reference to reported experimental values was found suitable for the screening of complex chemical systems for possibly relevant reactions. In particular, potentially relevant reactions can be singled out and scrutinized in detail when prioritizing chemicals for environmental risk assessment.
\end{abstract}

\section{Introduction}

Free radicals are produced (photo)chemically in living cells, surface water, electrochemical devices and many other systems. A significant number of free radicals are reactive and oxidize molecules via diverse reaction pathways. They are important in the context of electrochemistry, ${ }^{1}$ environmental science ${ }^{2}$ and biology, ${ }^{3}$ and there is an interest to understand reaction pathways and to quantify the rates of reaction. Experimental methods to determine the rate constant, $k_{\mathrm{r}}$, of a chemical reaction are in general laborious. ${ }^{4}$ Besides the cost of instrumentation and lab space, measuring a single rate constant with proper controls and data evaluation easily takes a skilled experimenter 3 days.

With $100000+$ chemicals to be assessed, ${ }^{5,6}$ there is a growing need to supplement experiments with computational methods to

\footnotetext{
${ }^{a}$ Eidgenössische Technische Hochschule (ETH) Zurich, Laboratory of Inorganic Chemistry, Vladimir-Prelog-Weg 1, 8093 Zurich, Switzerland.

E-mail: tom.m.nolte@gmail.com,noltet@inorg.chem.ethz.ch,tom.nolte@psi.ch, t.nolte@science.ru.nl

${ }^{b}$ Department of Environmental Science, Institute for Water and Wetland Research, Radboud University Nijmegen, 6500 GL Nijmegen, The Netherlands

${ }^{c}$ Electrochemistry Laboratory, Paul Scherrer Institut, 5232 Villigen PSI, Switzerland

${ }^{d}$ National Institute of Public Health and the Environment, PO Box 1,

3720 BA Bilthoven, The Netherlands

${ }^{e}$ Institute of Environmental Sciences (CML), Leiden University, PO Box 9518,

2300 RA, Leiden, The Netherlands

$\dagger$ Electronic supplementary information (ESI) available. See DOI: 10.1039/d0cp03750h
}

reduce costs and increase the sample variation and throughput. ${ }^{7-9}$ With this large number of chemicals, not all the reaction rate constants and pathways can be calculated with ultimate precision. Instead, we need 'cheap' calculus methods which are accurate and precise enough to (1) determine the rate constants of the bulk of reactions and (2) identify those reactions that require further, more detailed and expensive scrutiny.

Prediction of $k_{\mathrm{r}}$ is possible via, for example, 'machine learning' approaches which use a variety of algorithms and easily retrievable topological, electrostatic, and energetic parameters. ${ }^{2}$ The approaches yield relationships of mixed quality that depend on the type of radical and substrate considered. Many machine learning methods are 'black box' approaches, not supporting facile interpretation in terms of reaction mechanisms. In addition, parameters can be ill-defined for molecules that are complex or outside the model's domain of applicability. ${ }^{10-12}$ A different line of research aims to predict $k_{\mathrm{r}}$ mechanistically. 'Ab initio' methods base calculations of $k_{\mathrm{r}}$ on an accurate and precise determination of the free energy of activation $\Delta G^{\ddagger}$. The rate constant, $k_{\mathrm{r}}$, of a chemical reaction is described by the Eyring-equation as ${ }^{13}$

$$
k_{\mathrm{r}}=\frac{k T}{h} \times q \times \mathrm{e}^{\left(-\Delta G^{\ddagger} / R T\right)}
$$

$\Delta G^{\ddagger}$ is the free energy of activation, $R$ the universal gas constant, $T$ the absolute temperature and $q$ a statistical factor. The $a b$ initio 
methods involve the exploration of free energy changes along the reaction coordinate but the time-dependent three-dimensional interactions involved take much computing power for a full description. ${ }^{14}$ Simplifications might use static configurations for reactants or transition states, but require foreknowledge on the reaction pathway. Moreover, the statistical factor $q$ (eqn (1)) has to be described separately; $q$ differs between non-spherical 'complex' reactants. ${ }^{15}$

Simplifications of the Eyring-equation are free-energy relationships, made famous by the work of Hammett and Taft. ${ }^{16,17}$ For reactions with a closely similar rate-determining step, LFER stipulates that differences in Gibbs energy of activation, or the ratio of rate constants $k_{\mathrm{r} 1} / k_{\mathrm{r} 2}$ for two substrates 1 and 2 , are proportional to the difference in the reaction free energy change $\Delta G_{\mathrm{r}}$, eqn (2) and Fig. 1 :

$$
\log \left(\frac{k_{\mathrm{r} 1}}{k_{\mathrm{r} 2}}\right) \approx \Delta\left(\Delta G^{\ddagger}\right) \approx \sigma_{\mathrm{LFER}} \Delta\left(\Delta G_{\mathrm{r}}\right)
$$

where $\sigma_{\text {LFER }}$ is the substituent (fitting) constants. ${ }^{16,18}$ This approach has been successful because it uses semi-empirical parameters, hence requires less effort as compared to $a b$ initio methods. Thermodynamic values, such as the Gibbs energies of reaction $\Delta G_{\mathrm{r}}$, are often available from literature or comparatively easy to estimate computationally and are, therefore, 'cheap'.

LFER predictions are possible for reactions of chemicals with conventional, 'non-exotic', substituents ${ }^{19}$ and when $\sigma_{\text {LFER }}$ values are available (Fig. 1). Many LFERs developed over the years predict $\Delta\left(\Delta G^{\ddagger}\right)$ with reasonable accuracy. Comparison of $\Delta\left(\Delta G_{\mathrm{r}}\right)$ with experimental data allowed to approximate $\sigma_{\text {LFER }}$ for various radicals (e.g. $\mathrm{O}_{2}^{\bullet-} / \mathrm{HOO}^{\bullet}$ and aryl radicals ${ }^{20,21}$ ). Curiously, for those radicals, $\sigma_{\text {LFER }}$ was identical (i.e. within 2 standard deviations). Given these findings, ${ }^{18,20-22}$ we hypothesized $\sigma_{\text {LFER }}$ to be a constant factor for a broader set of radicals.

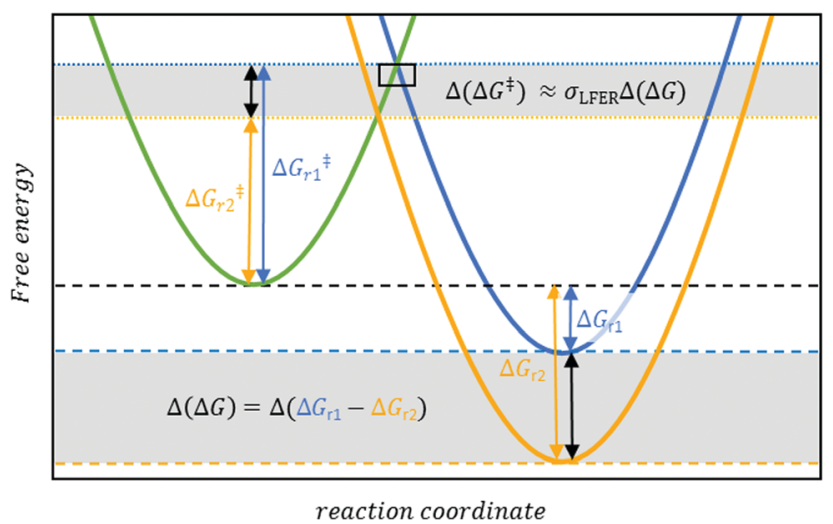

Fig. 1 Linear free energy relationships establish that, in a first approximation, the difference in activation energies $\Delta\left(\Delta G^{\ddagger}\right)$ (dotted lines) is proportional ( $\sigma_{\text {LFER }}$ as the proportionally constant) to the differences in Gibbs energies $\Delta(\Delta G)$. (dashed lines) between reactions, e.g. $r 1$ (blue curve) and $r 2$ (yellow curve). The concept ${ }^{16}$ offers the prospect of efficient computational methods to predict $\Delta G^{\ddagger}$ and, by extension, $k_{r}$. The rectangle symbol at the intercept between green and blue curves refers to Fig. 2 .
This implies that all radical-induced one-electron transfers have a similar rate-determining step.

The prediction of $\Delta\left(\Delta G^{\ddagger}\right)$ via LFER is a successful, albeit crude estimation, eqn (2). Changing solvents, i.e. different dielectric constants of the environment, is for instance known to alter product distribution and reaction kinetics. ${ }^{23}$ This is direct evidence that charge polarization in the transition state (TS) can affect the Gibbs energies of activation for a specific transition state, and thus promote/inhibit a specific reaction pathway. In other words, there are additional, usually smaller, contributions to the activation energy, $\Delta\left(\Delta G_{\mathrm{TS}}^{\ddagger}\right)$, that we need to consider, ${ }^{24}$ eqn (3):

$$
\Delta\left(\Delta G^{\ddagger}\right)=\sigma_{\mathrm{LFER}} \Delta\left(\Delta G_{\mathrm{r}}\right)+\Delta\left(\Delta G_{\mathrm{TS}}^{\ddagger}\right)
$$

Thus, the practical problem is the development of computationally efficient calculus of the quantum chemical influences on the transition states hidden in the term $\Delta\left(\Delta G_{\mathrm{TS}}^{\ddagger}\right)$, Fig. 2:

We therefore chose to calculate rate constants with a modified LFER approach that combines the 'cheap' calculus of the free-energy of reaction with selective ' $a b$ initio'-style additions. The selection reflects an analysis of over 500 published reactions of hydroxyl radicals. The resulting factors governing the rate constants originally caused us to embrace LFER terminology for the explanation of our work.

In our study, we investigated the details in transition states for one-electron oxidations, i.e. reactions that involve the net transfer of a single electron and, possibly, a single proton. We explain our selection of easily accessible parameters to successfully calculate and predict $\Delta\left(\Delta G_{\mathrm{TS}}^{\ddagger}\right)$. With it, we estimate rate constants for one-electron transfer, or radical oxidation, reactions.

\section{Methods}

Linear free-energy relationships (LFERs) assume that $\Delta\left(\Delta G_{\mathrm{r}}\right)$ relates to $k_{\mathrm{r} 1} / k_{\mathrm{r} 2}$ under the condition of an 'identical' mechanism, the same number of reacting sites and similar geometry. Eqn (2) allows for calculation of $\Delta\left(\Delta G^{\ddagger}\right)$ and $k_{\mathrm{r} 1} / k_{\mathrm{r} 2}$ based on knowledge of $\Delta\left(\Delta G_{\mathrm{r}}\right)$.

For a successful use of LFER, all parameters for the basic eqn (2), prominently the proportionality constant $\sigma_{\text {LFER }}$, need to be derived. Our parametrization process, together with the introduction of all the modifications $\left(\Delta\left(\Delta G_{\mathrm{TS}}^{\ddagger}\right)\right.$ and the statistical factor $q$, eqn (1) and (3), respectively), is described step by step in the Sections 2.1-2.3.

The parametrization was developed with known reactions of $\mathrm{HO}^{\bullet}$ radicals, a case with ample experimental data (ESI, $\dagger \mathrm{S} 1$ ), and opting for a model with a minimal set of parameters that would allow for 'cheap' and reliable calculation of unknown rate constants. We chose to calculate the Gibbs energy of reaction, $\Delta G_{\mathrm{r}}$, as per reactive site, which ensures the same number of reacting sites in the LFER (i.e. one). The total reactivity of the molecule is then calculated as the sum of the reactivities of all individual $\mathrm{R}-\mathrm{H}$ bonds.

Subsequently, the approach as outlined above was validated with data from reactions of $\mathrm{HOO}^{\bullet}, \mathrm{H}^{\bullet}$ and $\mathrm{CH}_{3}{ }^{\bullet}$ (see Results section). 


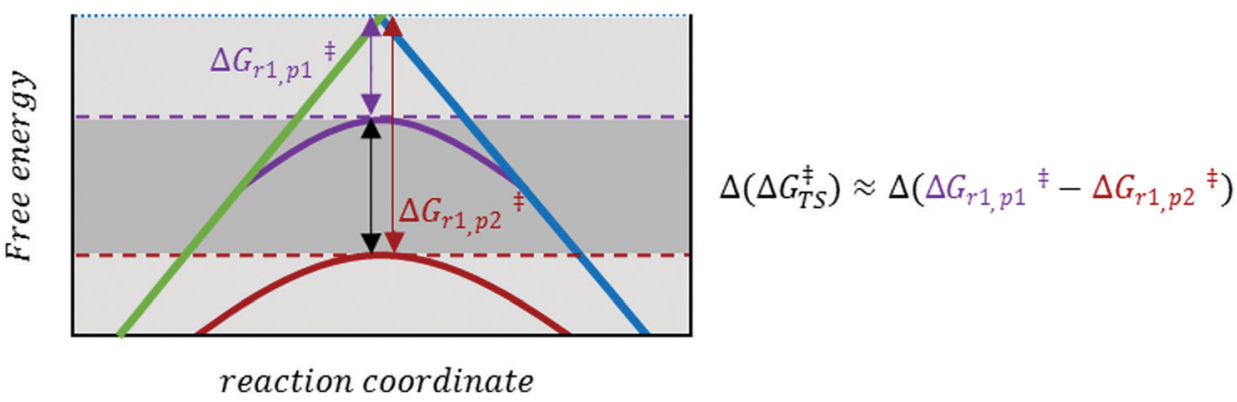

Fig. 2 Enlarged version of the black rectangle in Fig. 1. Depending on the nature and environment of the reaction site, a specific pathway (p1 or p2, indicated by purple and red curves, respectively) may be favoured. This is indicated by a 'perturbation' $\Delta(\Delta G \bar{i}$ s), of the 'normal' LFER behaviour (LFER depicted in Fig. 1).

In the validation process, the derived value for $\sigma_{\mathrm{LFER}}$ and the calculated values for $\Delta\left(\Delta G_{\mathrm{r}}\right)$ and $q$ were used to predict $k_{\mathrm{r} 1} / k_{\mathrm{r} 2}$, which in turn was compared to experimental data.

\subsection{Gibbs energy calculations: $\Delta G_{\mathbf{r}}$}

Eqn (2) predicts that prominent influences on $\Delta\left(\Delta G^{\ddagger}\right)$ are the Gibbs energy of the reaction $\Delta\left(\Delta G_{\mathrm{r}}\right)$ and the scaling factor $\sigma_{\text {LFER }}$. Empirically, we used values for $\Delta\left(\Delta G^{\ddagger}\right)$ for one-electron oxidation reactions (e.g. blue and yellow in Fig. 1) to quantify the relevance of $\Delta\left(\Delta G_{\mathrm{r}}\right)$. Thereby, we also determined $\sigma_{\mathrm{LFER}}$. As we calculated the values for $\Delta\left(\Delta G^{\ddagger}\right)$ per reaction site, they do not include the statistical factor (Section 2.3).

Perturbation theory leads to a formula to calculate $\Delta G_{\mathrm{r}}$ based on 'frontier orbital' interaction and charge transfer. ${ }^{25}$ In its simplified form (S3, ESI $\dagger$ ), the reaction free energy change for oxidation reactions is described as:

$$
\Delta G_{\mathrm{r}}=\Delta G_{\mathrm{ET}, \mathrm{ox}}-\Delta G_{\mathrm{CT}}=\Delta G_{\mathrm{ET}, \mathrm{ox}}-\Delta G_{\mathrm{PT}}
$$

with possible driving forces being oxidative electron transfer (ET,ox) and additional "charge transfer" (CT), e.g. proton-transfer (PT). The reactions considered do not imply a change in net charge. Therefore, eqn (4) reflects negligible changes in the Gibbs-energy of solvation.

Any parametrization routine starts with the parameters of large influence and subsequently proceeds those with smaller impact. The most prominent contributions to the activation energy, $\Delta G^{\ddagger}$, are correlated to ET and PT, i.e. their contributions are most influential. By definition, no reactions occur without electrons. Hence, we began with the calculation of the contribution of ET.

2.1.1. Transfer of the electron. The $\Delta G$ for ET relates to interactions between the highest occupied molecular orbital (HOMO) and the lowest unoccupied molecular orbital (LUMO) of the closed-shell molecule $\mathrm{R}^{-}$and the singly occupied molecular orbital (SOMO) of the radical $\mathrm{X}^{\bullet}$, the so-called "frontier orbitals". ${ }^{26}$ Outer-sphere ET is frontier-controlled (energetically and spatially) and does not involve charge-transfer complexes. ${ }^{27-29}$ We describe oxidative ET via the energy difference in the frontier orbitals, $E_{\mathrm{SOMO}}-E_{\text {номо }}$ as:

$$
\Delta G_{\mathrm{ET}, \mathrm{ox}} \propto \frac{1}{\left|E_{\mathrm{SOMO}, \mathrm{X}} \cdot-E_{\mathrm{HOMO}, \mathrm{R}^{-}}\right|}
$$

For computational details and associated uncertainties, we refer to the $\mathrm{ESI} \dagger(\mathrm{S} 2) \cdot E_{\text {номо }}$ (pure electron transfer) reflects the lower limit of the rate constant (Fig. S3A, ESI $\dagger$ and Fig. 6A). The majority of radical redox reactions are several orders of magnitude faster than that. In other words, the assumption of pure electron transfer often underestimates how favorable the reaction is, i.e. it overestimates the Gibbs activation energy of reaction.

2.1.2. Transfer of the proton. Compared to outer-sphere ET, hydrogen abstraction (HA) involves additionally the transfer of a positive charge not the result of the frontier electron (eqn (5) and eqn (S1), ESI + ). If a formal transfer of both an electron and a proton takes place, i.e. with proton-coupled electron transfer (PCET) or hydrogen atom transfer (HAT), energies of proton dissociation ( $\mathrm{p} K_{\mathrm{a}}$-values) need to be included. The overall result of the reaction does not involve charge transfer or even charge separation.

The homolytic bond dissociation enthalpy relates to the $\mathrm{p} K_{\mathrm{a}}$ of $\mathrm{R}-\mathrm{H}$ and the electron affinity of the radical $\mathrm{R}^{\bullet}{ }^{30-32}$ This applies to $\mathrm{X}-\mathrm{H}$ as well. The enthalpy change of a reaction between $\mathrm{R}-\mathrm{H}$ and $\mathrm{X}^{\bullet}$ involves an analysis of the relative bond strengths (blue rectangles in Fig. 3). Given the proton dissociation constants $\mathrm{p} K_{\mathrm{a}}(\mathrm{R}-\mathrm{H})$ and $\mathrm{p} K_{\mathrm{a}}(\mathrm{X}-\mathrm{H})$ of the conjugate hydrogen donor $\mathrm{X}-\mathrm{H}$ of the attacking radical $\mathrm{X}^{\bullet}$, respectively, eqn (6):

$$
E_{\mathrm{p} K}=2.3 R T\left[\mathrm{p} K_{\mathrm{a}}(\mathrm{R}-\mathrm{H})-\mathrm{p} K_{\mathrm{a}}(\mathrm{X}-\mathrm{H})\right]
$$

Frontier orbital ( $\left.E_{\text {Номо, eqn }}(5)\right)$ and charge transfer parameters $\left(\mathrm{p} K_{\mathrm{a}}\right)$ can, therefore, be combined into $\Delta G_{\mathrm{r}}$ :

$$
\Delta G_{\mathrm{r}} \propto \frac{1}{\left|E_{\mathrm{SOMO}, \mathrm{X}} \cdot-E_{\mathrm{HOMO}, \mathrm{R}^{-}}\right|-E_{\mathrm{p} K}}
$$

If the $\mathrm{p} K_{\mathrm{a}}$ 's of $\mathrm{R}-\mathrm{H}$ and $\mathrm{X}-\mathrm{H}$ are equal, we return to eqn (5) (pure $\mathrm{ET})$. We obtained $\mathrm{p} K_{\mathrm{a}}$ values from the literature and, when unavailable, by regression with atomic charges (S2, ESI $\dagger$ ). The thermodynamic cycle in Fig. 3 reflects graphically the calculation of $\Delta G_{\mathrm{r}}$ values for HA:

\subsection{Quantum chemical corrections}

In the Hammett/Taft equation (eqn (2)), $\sigma_{\mathrm{LFER}}$ is introduced to compare between congeneric chemicals, i.e. chemicals with 'the same' reaction mechanism, which we know is not the case for the reactions we include here: an electron transfer is not a hydrogen abstraction. In order to apply the LFER assumption, we need a correction for different mechanisms (purple and red in Fig. 2). Therefore, we chose to include the parameter $\Delta\left(\Delta G_{\mathrm{TS}}^{\ddagger}\right)$ 


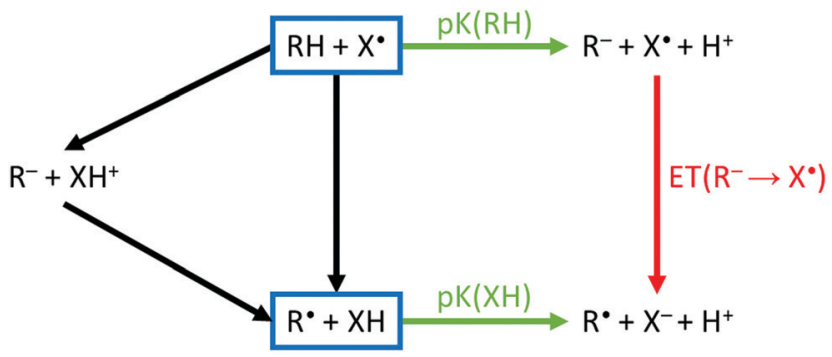

Fig. 3 Thermochemical cycle for $\mathrm{H}$-abstraction. The reaction driving force for both sequential (left) or concerted (center) mechanisms is described using proton transfer ( $\mathrm{p} K_{\mathrm{a}}$, green arrows) and electron transfer (red arrow). Electron transfer in absence of protolysis (black). The colors of arrows correspond to the data in Fig. S3 (ESI $\dagger$ ). Depending on the energies of proton/electron-transfer, an ionic or a non-ionic transition state is preferred, and the mechanism is sequential or concerted.

into eqn (3) with the assumption that $\Delta\left(\Delta G_{\mathrm{TS}}^{\ddagger}\right)$ is distinctly smaller than $\sigma_{\mathrm{LFER}} \Delta\left(\Delta G_{\mathrm{r}}\right)$.

2.2.1. Transition state stabilization: $\Delta\left(\Delta G_{\mathrm{TS}}^{*}\right)$. In order to obtain 'experimental' values for $\Delta\left(\Delta G_{\mathrm{TS}}^{\ddagger}\right)$ we compared our values for $\sigma_{\text {LFER }} \Delta\left(\Delta G_{\mathrm{r}}\right)$ obtained above with $\Delta\left(\Delta G^{\ddagger}\right)$, in eqn (7a):

$$
\begin{aligned}
& \Delta\left(\Delta G_{\mathrm{TS}}^{\ddagger}\right)=\Delta\left(\Delta G^{\ddagger}\right)-\sigma_{\mathrm{LFER}} \Delta\left(\Delta G_{\mathrm{r}}\right) \\
& \Delta\left(\Delta G_{\mathrm{TS}}^{\ddagger}\right)=\Delta\left(\Delta G^{\ddagger}\right)-\sigma_{\mathrm{LFER}} \frac{1}{\left|E_{\mathrm{SOMO}, \mathrm{X}^{\bullet}}-E_{\mathrm{HOMO} \mathrm{R}^{-}}\right|-E_{\mathrm{p} K}}
\end{aligned}
$$

with $E_{\mathrm{p} K}=2.3 R T\left[\mathrm{p} K_{\mathrm{a}}(\mathrm{R}-\mathrm{H})-\mathrm{p} K_{\mathrm{a}}(\mathrm{X}-\mathrm{H})\right]($ Section 2.2.2). Remember that $\Delta\left(\Delta G^{\ddagger}\right)$ relates back to Section 2.1 , eqn (7b):

$$
\Delta\left(\Delta G^{\ddagger}\right)=-\ln \frac{k_{\mathrm{p} 1}}{k_{\mathrm{p} 2}} / \frac{c_{\mathrm{HOMO}, \mathrm{p} 1}^{2} \Sigma_{\mathrm{p} 1} \sigma, \pi}{c_{\mathrm{HOMO}, \mathrm{p} 2}{ }^{2} \Sigma_{\mathrm{p} 2} \sigma, \pi}
$$

where $c_{\text {номо }}{ }^{2}$ and summation terms (eqn (8b)) characterize the statistical factor $q$ (Section 2.3).

We calibrated $\Delta\left(\Delta G_{\mathrm{TS}}^{\ddagger}\right)$ on ET and then introduced a correction for the involvement of protons, i.e. 'the polarization of the bond' in PCET and HAT. The so obtained range for $\Delta\left(\Delta G_{\mathrm{TS}}^{\ddagger}\right)$ involving reactions with $\mathrm{OH}^{\bullet}$ was $\sim 13 \mathrm{~kJ} \mathrm{~mol}^{-1}$.

2.2.2. Parametrization of $\Delta\left(\Delta G_{\mathrm{TS}}^{*}\right)$. Contributions for the TS required refinement. For the calculus, we parametrized $\Delta\left(\Delta G_{\mathrm{TS}}^{\ddagger}\right)$ using a computationally inexpensive method. The idea is to find a value that is proportional to the 'experimental' one and to derive the proportionality constant (here ' $z$ ', see below). Assuming $\Delta\left(\Delta G_{\mathrm{TS}}^{\ddagger}\right) \ll \sigma_{\mathrm{LFER}} \Delta\left(\Delta G_{\mathrm{r}}\right)$, its accuracy does not need to be as high as the latter, allowing for simplifications.

Charge polarization and solvent interactions in the TS depend on the mechanism (e.g. HAT, PCET), which depends on the type of H-donor involved. We distinguished types via atom-specific Fukui delocalizability indices. ${ }^{33}$ For computational details and associated uncertainties, we refer to S2 (ESI $\dagger$ ). The index, computed for the $\mathrm{R}-\mathrm{H}$ electron pair, can denote whether the H-atom has neighboring $\pi$-electrons $(\pi)$ or not $(\sigma)$. $\pi$-Transition states associate to allylic hydrogens $\left(\mathrm{sp}^{2}\right.$ hybridized centers) and $\sigma$-transition states to non-allylic hydrogens ( $\mathrm{sp}^{3}$ hybridized centers), Table T2 (ESI $\dagger$ ).

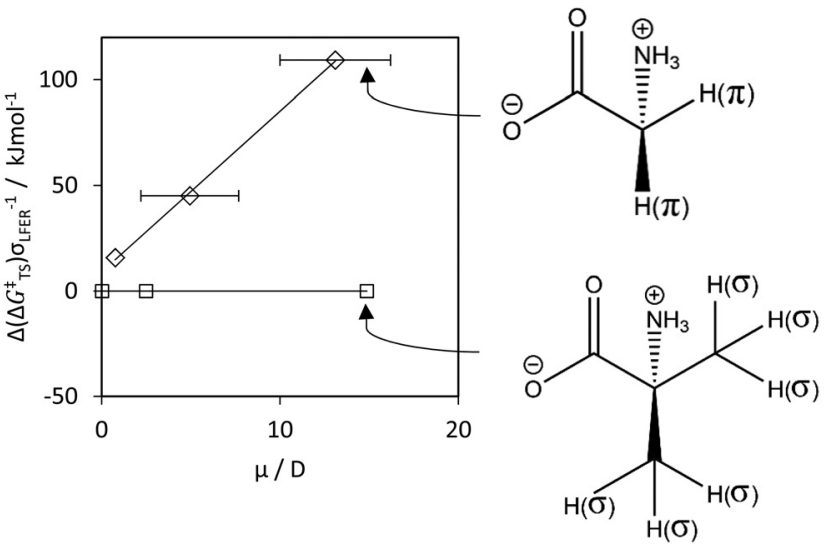

Fig. $4 \Delta\left(\Delta G\right.$ 节) for $\mathrm{HA}$ by $\mathrm{OH}^{\bullet}$ ( $y$-axis) versus the local dipole moment $\mu$ ( $x$-axis). We calculated $\Delta(\Delta G$ 辛s) via eqn (8), by taking the difference between 2 values: with $\mu$, and with $\mu=0$, i.e. $\Delta\left(\Delta G^{\#}\right.$ s $)=\Delta\left(\Delta G^{\ddagger}\right)-$ $\Delta\left(\Delta G^{\ddagger}(\mu=0)\right) . \Delta\left(\Delta G_{\mp}^{\ddagger}\right)$ characterizes the offsets between the LFERs in Fig. S $8 E(E S \mid \dagger)$. Diamonds and squares denote $\pi$ - and $\sigma$-type hydrogens, respectively solid lines denote data fits. We used the fit to parametrize the contribution by $\mu$ to $\Delta G^{\ddagger}$. Molecules illustrate the combined impact of $\sigma, \pi$ and $\mu$ on $\Delta\left(\Delta G_{F}^{*}\right.$ S).

To parametrize specific TS effects, we introduced a global scaling parameter $z$. We calculated the correction for the transition state stabilization with $z_{\sigma}=0$ for $\pi$-transition states and $z_{\pi}=0$ for $\sigma$-transition states, eqn (8):

$$
\begin{gathered}
\Delta\left(\Delta G_{\mathrm{TS}, \sigma}^{\dot{亠}}\right)=-z_{\sigma} \\
\Delta\left(\Delta G_{\mathrm{TS}, \pi}^{\ddagger}\right)=-z_{\pi} \mu
\end{gathered}
$$

In eqn (7), $\mu$ is the local dipole moment surrounding $\mathrm{R}-\mathrm{H}$ and $z$ was left for radical-specific empirical fitting. The slope of the lines in Fig. 4 is the scaling parameter $z$, denoting the strength of the proportionality between $\Delta\left(\Delta G_{\mathrm{TS}}^{\ddagger}\right)$ and the local dipole moment within the molecule. Note that if either $z_{\sigma}=\mu=0$ or $z_{\pi}=0, \Delta\left(\Delta G_{\mathrm{TS}}^{\ddagger}\right) \approx 0$ (Fig. 4). In this case, $\Delta G^{\ddagger}$ is directly proportional to $\Delta G$ (which follows from eqn (2)). As the dipole moment is tied to solvation free energies, the proportionality characterizes charge polarization in the TS.

\subsection{The statistical factor $q$}

In the Eyring equation (eqn (1)) $q$ is a statistical factor related to the reaction probability. Following eqn (8), we treated $q$ as a product function:

$$
q=q_{\Sigma(\pi, \sigma)} q_{C \mathrm{HOMO}(\pi, \sigma)^{2}}
$$

$q$ prominently includes the number of abstraction sites $\Sigma(\pi, \sigma)$, Fig. S9-2 (ESI $\dagger$ ). For compounds with only equivalent $\mathrm{H}$ atoms, i.e. symmetric molecules, we can use simple corrections based on symmetry. ${ }^{19}$ We derived the expected values for $k_{\mathrm{r}}$ depending on $\sigma \Delta G_{\mathrm{r}}$ and the reaction pathway (TS effects, in Section 2.2). We filtered the data used for parametrization based on symmetry (see Table T2, ESI $\dagger$ ) and then express $q_{\Sigma(\pi, \sigma)}$ 

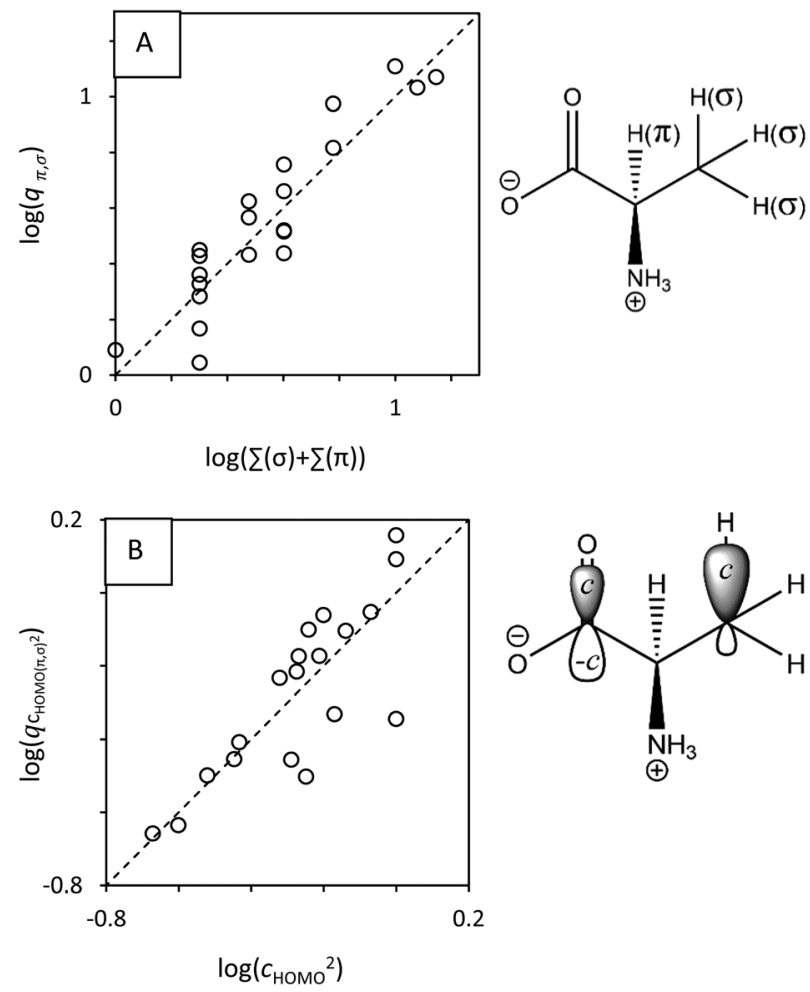

Fig. 5 (A) The statistical factor $q$ (on the $y$-axis), calculated using experimental data and eqn (8), is the number of equivalent $\mathrm{H}$ atoms, wherein $\pi$ and $\sigma$ denote the presence of either resonance stabilized or nonresonance stabilized mechanisms (which do not have neighboring $\pi$-electrons), respectively. The molecule illustrates $\pi$ and $\sigma$ : there are 3 equivalent $\sigma$ hydrogens; $1 \pi$ hydrogen. (B) The statistical factor $q$ (on the $y$-axes), calculated using experimental data and eqn (8). $q$ correlates with the squared atomic orbital coefficient. The dashed line denotes theoretical relationships for symmetric molecules.

for the resulting compounds as:

$$
q_{\Sigma(\pi, \sigma)}=\frac{k_{\mathrm{r}, \mathrm{observed}}}{q_{\left.C_{\mathrm{HOMO}(\pi, \sigma)}\right)^{2}} \cdot k_{\mathrm{r},(\mathrm{R}-\mathrm{H})}}
$$

wherein the denominator is the expected rate constant for a single R-H bond $(\pi=\sigma=1)$. We can see that the values for $q_{\Sigma(\pi, \sigma)}$ vary by a factor 10 , Fig. $5 \mathrm{~A}$. We thus used the number of 'chemically equal' hydrogen atoms $\Sigma(\pi, \sigma)$ to account for this, Fig. 5A:

$q$ can also be understood as the accessibility of abstraction sites, i.e. it comprises also the 'effectiveness' of collisions, the so-called quantum-mechanical 'transmission'. By extension, the overlap between frontier orbitals is characteristic of the space in which the electron can move within the TS. ${ }^{26,34}$ Thus, we can express $q_{C \text { Номо }(\pi, \sigma)^{2}}$ as function of squared $\mathrm{AO}$ coefficients, $c_{\mathrm{HOMO}}{ }^{2}$ (Fig. $5 \mathrm{~B}$ ):

$$
q_{C_{\mathrm{HOMO}(\pi, \sigma)}{ }^{2}}=\frac{k_{\mathrm{r}, \mathrm{observed}}}{q_{\Sigma(\pi, \sigma)} \cdot k_{\mathrm{r},(\mathrm{R}-\mathrm{H})}}
$$

wherein the numerator is the expected rate constant for the molecule reflecting similar-sized orbitals $\left(c_{\mathrm{HOMO}(\pi, \sigma)^{2}}=1\right)$.
We then see that the values for $q_{C \mathrm{HOMO}(\pi, \sigma)^{2}}$ vary by almost a factor 10, Fig. 5B.

We obtained atomic orbital (AO) coefficients (denoted as

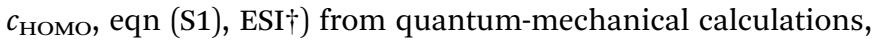
denoting the 'size' or 'shape' of orbitals (Fig. 5B). Computational details and associated uncertainties are provided in S2 (ESI $\dagger)$. We thereby used the data for congeneric H-donors, to take into account the dependence of $q_{C \mathrm{HOMO}(\pi, \sigma)^{2}}$ on $\mathrm{AO}$ coefficients, Fig. 5B.

\section{Results}

\subsection{Linear free energy relationships}

Within the (crude) model of LFER only (eqn (2)), $\Delta G_{\mathrm{r}}$ can predict $\sim 95 \%$ of the $k_{\mathrm{r}}$ values $(N \approx 500)$ within \pm 1 order of magnitude of the expected value (Fig. S3B, ESI, $\dagger p<1 \times 10^{-5}$ ), i.e. for these $95 \%$, the estimation of the activation energy $\Delta G^{\ddagger}$ was accurate within $6 \mathrm{~kJ} \mathrm{~mol}^{-1}$.

Using symmetry-filtered data (Section 2.1), we found $\sigma_{\text {LFER }}=$ $\log \left(k_{\mathrm{r}}\right) / \Delta G_{\mathrm{r}}=2.3 \mathrm{eV}^{-1}$, i.e. the rate constant doubles if the Gibbs energy of the reaction decreases by $13 \mathrm{~kJ} \mathrm{~mol}^{-1}$. We obtained $\sigma_{\text {LFER }}$ values $( \pm 2 \mathrm{SD})$ of $2.4( \pm 0.2)$ and $2.1( \pm 0.2) p\left(k_{\mathrm{r}}\right) / \mathrm{eV}$ for $\mathrm{H}^{\bullet}$ (Fig. 8) and $2.6( \pm 0.6) p\left(k_{\mathrm{r}}\right) / \mathrm{eV}$ for $\mathrm{CH}_{3}{ }^{\bullet}$ (Fig. S12, $\mathrm{ESI} \dagger$ ). These values do no statistically differ from those for $\mathrm{OH}^{\bullet}$ or $\mathrm{OOH}^{\bullet} / \mathrm{O}_{2}^{\bullet-21}$ (within two SDs).

\subsection{Transition state stabilization: $\Delta\left(\Delta G_{\mathrm{TS}}^{\ddagger}\right)$}

For $\pi$-type systems, our calculated $\Delta\left(\Delta G^{\ddagger}\right)$ shows a systematic deviation from the known rate data for water that is dependent on the dipole moment $\mu$. In other words, hydrogen abstraction from non-polar hydrogen donors shows lower $\Delta\left(\Delta G_{\mathrm{TS}}^{\ddagger}\right)$ compared to HA from polar and zwitterionic $\pi$-type hydrogen donors, Fig. 5 . Parametrization of this effect resulted in a satisfactory fit of the rate constants, Fig. 6C.

As a validation, we used the parametrized result to predict $k_{\mathrm{r}}$. for $\mathrm{OOH}^{\bullet}, \mathrm{H}^{\bullet}$ and $\mathrm{CH}_{3}{ }^{\bullet}$. Aqueous-phase abstraction by $\mathrm{H}^{\bullet}$ of a $\pi$-type $\mathrm{H}$ atom from a zwitterionic compound is $\approx 200$ times slower ( $\approx 2.3$ in log units, Fig. 7) than for a $\sigma$-type $\mathrm{H}$ atom. The difference in terms of $\Delta\left(\Delta G^{\ddagger}\right)$ is $\approx 13 \mathrm{~kJ} \mathrm{~mol}^{-1}\left(\approx 100 \mathrm{~kJ} \mathrm{~mol}^{-1}\right.$ in terms of $\left.\Delta\left(\Delta G_{\mathrm{r}}\right)\right)$. The values match those for $\Delta\left(\Delta G_{\mathrm{TS}}^{\ddagger}\right)$ for HA involving $\mathrm{OH}^{\bullet}$ (Fig. S8E, ESI $\dagger$ ) and $\mathrm{CH}_{3}{ }^{\bullet}$ (Fig. S12A, ESI $\dagger$ ). However, propene (purple star in Fig. 7) did not fit our prediction (see Discussion, Section 4.3.1). S4 (ESI $\dagger$ ) provides additional controls.

\subsection{The influence of radical electron affinity $e_{x}$}

As explained above, we developed our method with reactions of $\mathrm{OH}^{\bullet}$ radicals, where ample data is available. The predictions were very satisfactory. However, when we extended our database with controls for $\mathrm{HOO}^{\bullet}, \mathrm{H}^{\bullet}$, and $\mathrm{CH}_{3}{ }^{\bullet}$ (Tables T2-T5, $\mathrm{ESI} \dagger$ ), some minor systematic, radical-specific deviations from prediction became evident.

Eqn (8) contains 2 radical-specific properties: $\mathrm{p} K_{\mathrm{a}}(\mathrm{X}-\mathrm{H})$ and $E_{\mathrm{Somo}, \mathrm{X}^{*}}$. The error, therefore, is introduced in one of the two values. As we used the experimentally determined value 

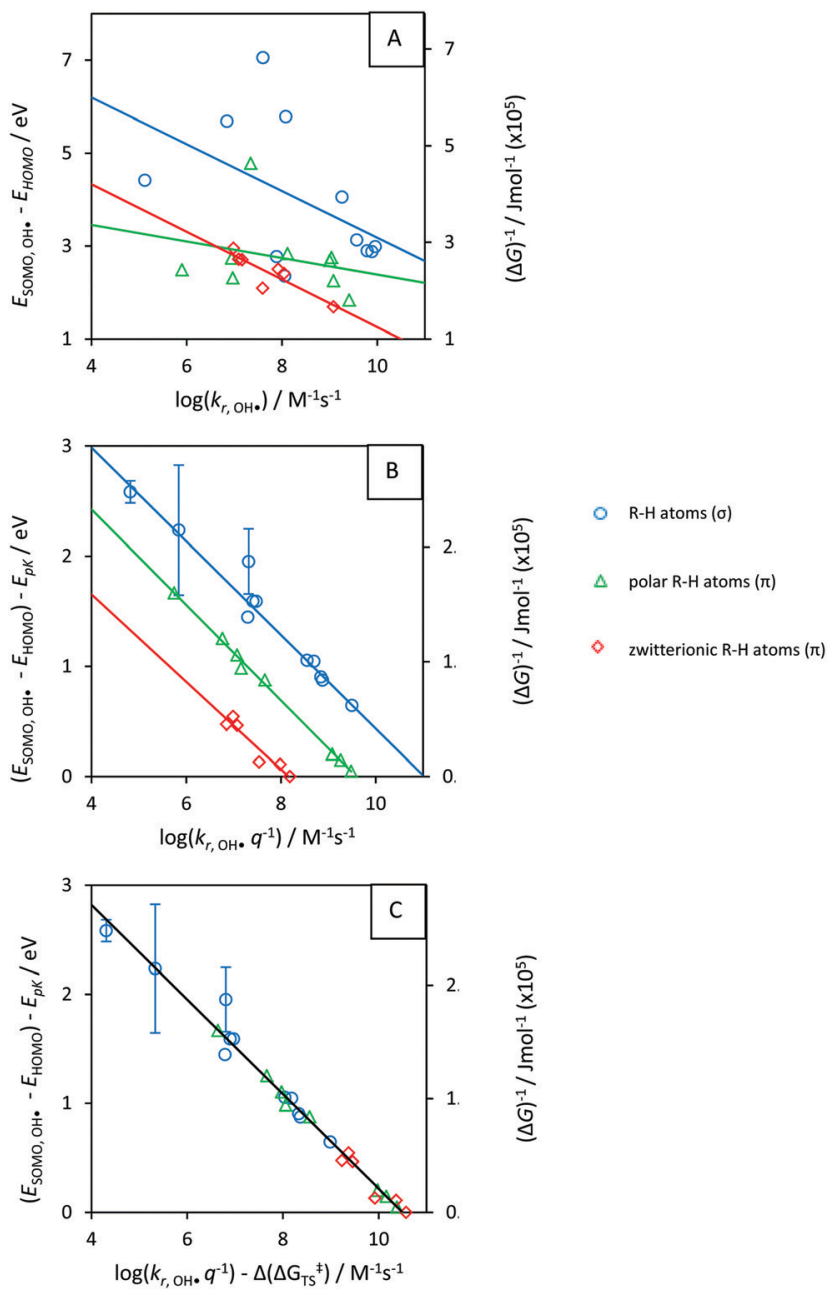

Fig. 6 Rate constants $\left(k_{r}\right)$ for reaction with $\mathrm{OH}^{\bullet}(x)$, versus reaction energies (y). (A) $k_{r}$ versus frontier orbital energies; (B) $\Delta G^{\ddagger}$ versus $\Delta G_{r}$; (C) $\Delta G^{\ddagger}-\Delta\left(\Delta G^{\ddagger} s\right)$ versus $\Delta G_{r}$. Only symmetric compounds (with equivalent $\mathrm{R}-\mathrm{H}$ groups) are taken into account. Energies were converted using $100 \mathrm{~kJ} \mathrm{~mol}{ }^{-1} \hat{=} 1.04 \mathrm{eV}$ and $0 \mathrm{~kJ} \hat{=}-8 \mathrm{eV} \approx E_{\mathrm{SOMO}}\left(\mathrm{OH}^{\bullet}\right)$. Lines are data fits. Error bars indicate uncertainty from predicted $\mathrm{p} K_{\mathrm{a}}$ (Chemaxon program ${ }^{35}$ ). Full parametrization in Fig. S8 and Table T2 (ESI $\dagger$ ).

of $\mathrm{p} K_{\mathrm{a}}(\mathrm{X}-\mathrm{H})$ for $\mathrm{HOO}^{\bullet}, \mathrm{H}^{\bullet}$ and $\mathrm{HO}^{\bullet}$, we assume the error to relate to our calculation of $E_{\mathrm{SOMO}, \mathrm{X}^{\bullet}}(\mathrm{S} 2, \mathrm{ESI} \dagger)$. We then decided to derive experimental values for $E_{\mathrm{SOMO}, \mathrm{x}^{*}}$ (via fitting) and obtained an 'electron affinity', $\mathrm{e}_{\mathrm{x}}$. If we compare the two values, Fig. 8, they are identical for the $\mathrm{OH}^{\bullet}$ radical: $\mathrm{e}_{\mathrm{OH}^{\bullet}}=8.0( \pm 0.5) \approx$ $E_{\mathrm{SOMO}_{\mathrm{OH}}}{ }^{*}=8.0( \pm 0.2) \mathrm{eV}$. For best intercomparison between radicals, $E_{\mathrm{SOMO}, \mathrm{X}^{*}}$ has to be replaced with $\mathrm{e}_{\mathrm{x}}$ in eqn (8):

\section{Discussion}

With our method for calculus, we can estimate rate constants of one-electron oxidation processes with a statistical reproducibility of $R^{2}=0.98$ and with a confidence $( \pm 1 \mathrm{SD}$, i.e. $68 \%)$ of $30-40 \%$. In other words, the activation energy is predicted with an accuracy of around $1 \mathrm{~kJ} \mathrm{~mol}^{-1}$. We want to compare the result to errors from experimental data: rate data for fast kinetics with

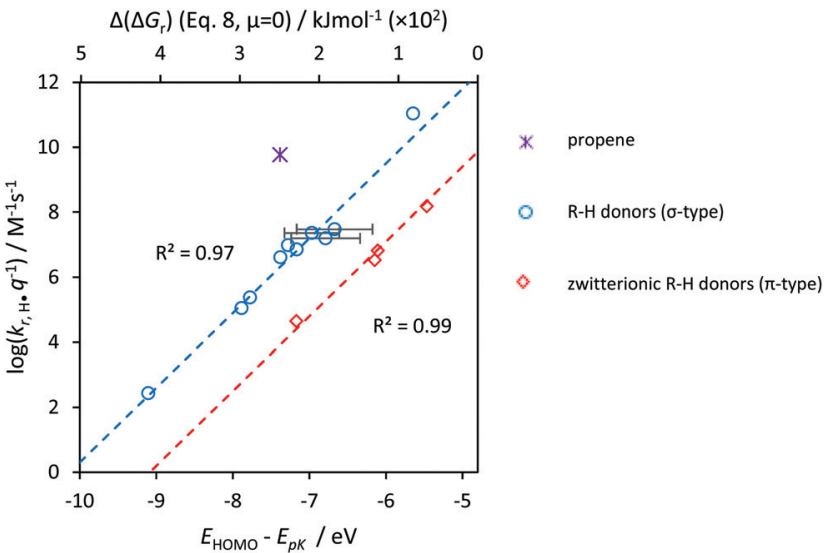

Fig. 7 The product of the rate constant $\left(k_{r}\right)$ and the statistical factor $q$, logarithmically transformed, for reaction with $H^{\bullet}(y)$ versus $\Delta G_{r}(x)$. Dashed lines denote the LFERs. Red diamonds are $\pi$-type zwitterionic $\mathrm{R}-\mathrm{H}$ and blue circles are $\sigma$-type $\mathrm{R}-\mathrm{H}$. Error bars indicate uncertainty introduced upon prediction of $\mathrm{p} K_{\mathrm{a}}{ }^{35}$ Only compounds with equivalent $\mathrm{X}-\mathrm{H}$ groups are taken into account. The purple star is propene (outlier, discussed in

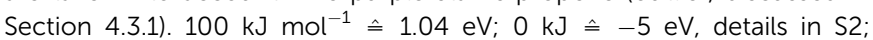
Table T3 (ESI†).

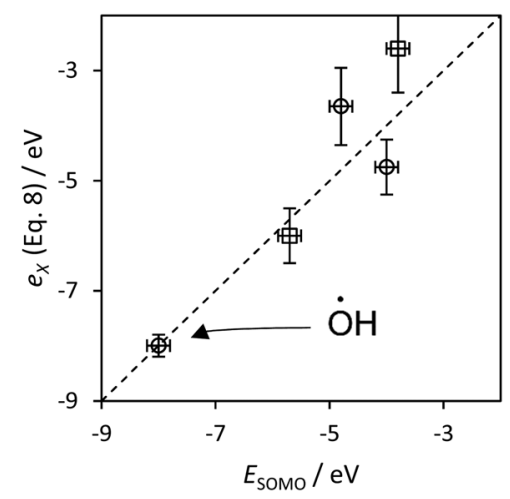

Fig. 8 Comparison of the electron affinity, $e_{x}$, with $E_{\text {SOмо }} \cdot x^{*}$. The dashed line denotes interaction between frontier orbitals allowing oxidative outersphere electron transfer. Data for $\mathrm{O}_{2}{ }^{\bullet-}$ (both abstraction and addition reactions) and $\mathrm{HOO}^{\circ} \mathrm{s}$ indicated by squared symbols were taken from ref. 21. $p<0.05$ (2SD).

rate constants $>10^{6} \mathrm{M}^{-1} \mathrm{~s}^{-1}$ are often reported with precisions better than $10 \%$. However, results of different labs routinely yield a distinctly higher scatter (20-40\%). One may assume, therefore, that the accuracy of data often cannot keep up with their reported precision. Our calculations have accuracies comparable to the accuracy of preliminary experiments.

Our method uses a minimal set of parameters to predict kinetic rate constants, though similar (but substantially larger) sets recur in both machine learning and $a b$ initio methods. Because of the minimization, parameters may embed more than 1 influence. However, for our examples and controls, the parametrization appears sufficient. Rather than quantifying explicit contributions to $\Delta\left(\Delta G^{\ddagger}\right)$ via 'expensive' ab initio methods, our parametrization is computationally 'cheap'. Calculations were carried out on a standard desktop computer and for the $\pm 40 \%$ 
prediction accuracy they took 5 seconds per molecule. Such a lowcost approach is very handy for selecting possibly relevant reactions in a complex reaction scheme. It enables, for example, large-scale screening of aquatic reactivity in environmental chemistry ${ }^{36,37}$ that can single out possibly relevant reaction pathways. This is the application for which the current method was developed. Then, in a second step, more accurate and precise data can be acquired either by experimental work, more sophisticated LFER-based methods, ${ }^{22,24,38,39}$ or even extensive $a b$ initio calculation.

We initially assumed that the influence of ET and PT would be largest, whereas other influences were assumed to be distinctly smaller. Statistical evaluation of data supports this notion (Fig. S10, ESI $\dagger$ ). Thus, our parametrization mainly relies on the assumption of a linear free-energy relationship between Gibbs energy of reaction and the Gibbs activation-energy of the same reaction. The former is given by the sum of the electron transfer and, if applicable, a proton transfer. We calculated the energies of electron and proton transfer via the energies of frontier orbitals and $\mathrm{p} K_{\mathrm{a}}$ values, respectively. We combined the terms via a thermodynamic cycle and Klopman's equation. $^{25}$

The exact values for activation energies were then refined by estimation of relative (de-)stabilization of transition states by local delocalization and polarization. This process is reiterated over all possible reactive sites of a molecule. The measured rate constant then is equal to the sum of all rate constants for individual reactive sites. Our initial hypothesis of a closely similar rate determining step for the one-electron oxidations by $\mathrm{HOO}^{\bullet}, \mathrm{H}^{\bullet}, \mathrm{CH}_{3}{ }^{\bullet}$ and $\mathrm{HO}^{\bullet}$ appears to be correct; our method is applicable for multiple radicals (Fig. 6-8 and Fig. S12, ESI $\dagger$ ).

\subsection{Statistical factor}

With symmetric molecules, inclusion of the statistical factor $q$ via $\Sigma(\sigma)+\Sigma(\pi)$ (see Fig. 3A) led to better correlations between $k_{\mathrm{r}}$ and $\Delta G$ between and within H-donor types (Fig. S8C compared to Fig. S8B, ESI $\dagger$ ). Similar findings were reported for unsaturated organics. ${ }^{40,41}$ The inclusion of $q$ via $c_{\text {номо }}$ (Fig. S8D, ESI $\dagger$ ) marginally improved the correlations (Fig. S10, ESI $\dagger$ ), whereas it slightly converged the offsets of the regressions for $\pi$-type $\mathrm{X}-\mathrm{H}$ compared to $\sigma$-type $\mathrm{X}-\mathrm{H}$ bonds (Fig. S8D compared to Fig. S8C, ESI $\dagger$ ). In contrast, the statistical factor $q$ (as described via $c_{\mathrm{HOMO}, \mathrm{R}^{-}}$, within a factor 5 ) did not improve the LFER for ET $(N=36$, Fig. S2 and Table T1, ESI $\dagger)$. Note that we can also interpret the influence of $c_{\text {номо }}$ in terms of $\Delta G_{\mathrm{r}}$ via Klopman's equation (eqn (S1), ESI $\dagger)^{25}$

If $q$ was included in the calculation model, the slopes of the LFERs (i.e. the substituent constants, $\sigma_{\text {LFER }}$ ) for $\mathrm{OH}^{\bullet}$ converged to $\approx 2.3 p\left(k_{\mathrm{r}}\right) / \mathrm{eV}$ (Fig. $6 \mathrm{~B}$ and Fig. S2, S8D (ESI $\dagger$ )). The slopes do not differ between $\pi$ and $\sigma \mathrm{H}$-atom types. Thus, $q$ improves the prediction accuracy of $k_{\mathrm{r}}$. The overall reaction rate constant is a combination of the accessibility of each individual hydrogen atom that can be abstracted (i.e. q), and depends on the reaction dynamics. ${ }^{42-44}$ Depending on the dynamics, symmetry arguments (Table $\mathrm{T} 2, \mathrm{ESI} \dagger$ ) may reduce the computational effort needed.

\subsection{Driving forces $\Delta G_{\mathrm{r}}$}

4.2.1. Electron transfer versus hydrogen abstraction. For electron transfer by $\mathrm{OH}^{\bullet}$ (eqn (2) and (3)), the $\sigma_{\text {LFER }}$ (Hammett substituent constant) value, $\pm 2 \mathrm{SD}$, is $2.2 \pm 0.2 p\left(k_{\mathrm{r}}\right) / \mathrm{eV}$, similar to that of $\mathrm{HOO}^{\bullet} / \mathrm{O}_{2}{ }^{--}\left(\sigma_{\text {LFER }}=2.1-2.5 p\left(k_{\mathrm{r}}\right) / \mathrm{eV}\right)$ including ET. ${ }^{21}$ Using a thermodynamic cycle (eqn (6)), we found that slopes of the LFERs $\left(\sigma_{\text {LFER }}\right)$ for $\mathrm{OH}^{\bullet}$ involving different types of $\mathrm{H}$-donors all converged to $\sim 2.3 p\left(k_{\mathrm{r}}\right) / \mathrm{eV}\left(0.024 p\left(k_{\mathrm{r}}\right) /\left(\mathrm{kJ} \mathrm{mol}^{-1}\right)\right)$. Thus, it appeared to us that the values for ET and HA do not significantly differ. For both, it implies doubling of the rate constant with every $-13 \mathrm{~kJ} \mathrm{~mol}^{-1}$ decrease of $\Delta G_{\mathrm{r}}$.

HA by $\mathrm{OH}^{\bullet}$ is usually faster than ET. ${ }^{45,46}$ Hence, the observed pathway is usually HA (similar for addition, Fig. S14, ESI $\dagger$ ). Our calculations give clear hints on the expected reaction mechanism. For example, the inclusion of ET only in the calculus may predict a very low rate constant as compared to the case of PCET/HAT. In that case, the contribution from proton-transfer is significant, and ET is not the prominent mechanism. In other words, the influence of $\mathrm{p} K_{\mathrm{a}}$ on the $\Delta G_{\mathrm{r}}$ values (eqn (6)) is non-negligible.

If experimental data are available, and if the calculated rate constant is significantly lower than the observed one, a misconception about the mechanism is to be expected. An obvious case is the reaction of the hydrogen atom with propene (outlier in Fig. 7). There, the observed reaction with $\mathrm{H}^{\bullet}$ is probably addition, ${ }^{47}$ not an oxidation (Tables T2-T5, ESI $\dagger$ ). Compared to $\mathrm{OH}^{\bullet}$ and $\mathrm{OOH}^{\bullet}$, which react as electron acceptors with most organic chemicals (Fig. S3B, ESI, $\dagger^{21}$ ), $\mathrm{H}^{\bullet}$ and $\mathrm{CH}_{3}{ }^{\bullet}$ might not (eqn (4)). Provided a correct assumption of mechanism, the applicability of the method is retained and eqn (8) can predict $k_{\mathrm{r}}$ for different radicals (Fig. 6-8).

The influence of $\mathrm{p} K_{\mathrm{a}}$ on the reaction rate (eqn (6)) can be interpreted as a Coulombic interaction between the hydrogen atom of $\mathrm{R}-\mathrm{H}$ and $\mathrm{X}^{\bullet}$, e.g. the oxygen atom of $\mathrm{OH}^{\bullet}$. A larger value for $Q_{\mathrm{H}}$ (lower $\mathrm{p} K_{\mathrm{a}}$ for $\mathrm{R}-\mathrm{H}$ ) implies greater electrostatic stabilization and higher $k_{\mathrm{r}}$. Provided that the solvent is inert, a higher $\mathrm{p} K_{\mathrm{a}}$ of $\mathrm{R}-\mathrm{H}$ implies a proportionally lower contribution of proton transfer to $\Delta G_{\mathrm{r}}$. (eqn (6)). Within the model of LFER, $\mathrm{HA}$ is expected to be faster than ET if the reaction is more favorable thermodynamically, i.e. the products including $\mathrm{H}_{2} \mathrm{O}$ (HA) are more stable than those including $\mathrm{OH}^{-}$(ET).

4.2.2. Comparison with other methods. Machine learning and $a b$ initio methods require separate models and simulations for individual chemicals. For example, charge-transfer interactions are described explicitly in ab initio methods, which consider charge transfer as a function of time and distance. Charge-based descriptors also recur in many QSPR models. ${ }^{2,48}$ However, proton transfer can also be characterized by static (cheap) descriptors; the use of $\mathrm{p} K_{\mathrm{a}}$ (Section 2.2.2) takes less computational effort.

LFERs for ET and HA exist for $\mathrm{OH}^{\bullet}, \mathrm{OOH}^{\bullet} / \mathrm{O}_{2}{ }^{\bullet-}, \mathrm{H}^{\bullet}$ and

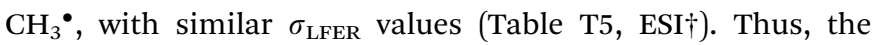
individual LFERs are not phenomenological, ${ }^{18}$ and the potential energy curves of the reactants can be described by a uniform method, e.g. a harmonic oscillator (depicted in Fig. 1) or Morse potential. In contrast, the relevance of $E_{\mathrm{HOMO}}$ and MoRSE potential $^{2,49,50}$ parameters and the QSPR model algorithm 
architecture differs between radical species. As MoRSE derives from molecular vibrational structure ${ }^{51,52}$ specification of potential curves $^{24,53}$ might facilitate extrapolation to a broader set of radicals.

The optima in $k_{\mathrm{r}}$ for $\mathrm{OH}^{\bullet}, \mathrm{OOH}^{\bullet}, \mathrm{H}^{\bullet}$ and $\mathrm{CH}_{3}{ }^{\bullet}$ generally correspond to cases when $\left|E_{\text {SOMO }}-E_{\text {HOMO }}\right| \rightarrow 0$ (Fig. 8); the reactivity sequence (Tables T2-T4, ESI $\dagger$ ) is in line with previous studies. $^{54-57} E_{\text {SOMO }}$ relates to electron accepting/donating ability, electrophilicity (S5, ESI $\dagger)^{54}$ and 'electron affinity', $\mathrm{e}_{\mathrm{x}}$ (Fig. 8 and Fig. S11, S12, ESI $\dagger$ ). ${ }^{54}$ We did not consider specific terms for orbital shapes of radicals (eqn (S1) and (S2), ESI $\dagger$ ) in the current study. Machine learning methods use associated terms (e.g. radial density function descriptors ${ }^{58,59}$ ). Hence, a direct description of orbital-orbital overlap and orbital symmetry factors may also characterize electron affinity and improve $\Delta G_{\text {r }}$ predictions.

\subsection{Transition state stabilization: $\Delta\left(\Delta G_{\mathrm{TS}}^{\ddagger}\right)$}

4.3.1. Proton-coupled electron transfer versus hydrogen atom transfer. The LFERs for HA were developed using a thermochemical cycle (Fig. 3) based on the assumption that the relative proton affinities of the reactants are not influenced by ET, and vice versa. In the case of a concerted mechanism, e.g. proton-coupled electron transfer (PCET), ${ }^{60,61}$ 'low' $\Delta G^{\ddagger}$ might be involved. Though at elevated temperature, abstraction of a ring and benzylic hydrogen dominate over addition, indicating larger $\Delta G^{\ddagger}$ values. $^{62}$ In such cases, sequential mechanisms might be involved. Indeed, Fig. $6 \mathrm{~B}$ and 7 illustrate the involvement of $\Delta\left(\Delta G_{\mathrm{TS}}^{\ddagger}\right)$ and the presence of mechanistically distinct pathways. In analogy, Pompe et al. predicted $k_{\mathrm{r}}$ based on differentiation between $\mathrm{C}-\mathrm{H}$ bond types. ${ }^{63,64}$

For H-donors with neighboring $\pi$-electrons ( $\pi$ in Fig. 5 ), $\mu_{\pi}$ positively correlates with $\Delta G^{\ddagger}$, eqn (8). This is because charge redistribution in the TS is involved. Abstraction of ' $\pi$-type hydrogens' normally occurs via proton transfer between the $\mathrm{R}-\mathrm{H}$ and $\mathrm{X}^{\bullet}$ ( $\sigma$-orbitals), but the electron transfers from the adjacent $\mathrm{sp}^{2}-\pi$ orbital (only atomic orbitals of the same symmetry can combine ${ }^{65}$ ). This often involves relatively high $\Delta G^{\ddagger}$. In analogy, captodative stabilization can influence $\Delta G^{\ddagger}$.

The rate is faster in $\mathrm{H}_{2} \mathrm{O}$ if the reaction involves movement of only 1 electron, rather than 3 electrons, or when the proton and electron are transferred between the same sets of orbitals ${ }^{66}$ (e.g. PCET reactions). This fits with the data for e.g. ammonia and 2-methylalanine (Table T2). These are polar and zwitterionic compounds, respectively, but $\mathrm{OH}^{\bullet}$ abstracts both the electron and proton via the $\sigma \mathrm{R}-\mathrm{H}$ bond (' $\sigma$-type' $\mathrm{H}$-atom) since there are no neighboring $\pi$-electrons. Hence their 'high' reactivity as compared to molecules containing polar and zwitterionic ' $\pi$-type' $\mathrm{H}$ atoms (Fig. S8E, ESI $\dagger$ ). The principles also apply to reactions involving pairs of electrons: in water, 2-electron reactions involving singlet oxygen $\left({ }^{1} \mathrm{O}_{2}\right)$ are faster than 4-electron reactions. ${ }^{49}$

4.3.2. Connecting mechanisms to thermodynamics. Reactivity depends on the relative probability and location of the electron transferred during the reaction, i.e. the reaction coordinate. For example, while the proton is abstracted from the $\sigma$-orbital, the $\sigma$-electron might delocalize into a $\mathrm{sp}^{2}-\pi$ system. In this case, the delocalization might be interpreted as an electronic coupling $^{67}$ or a third (intermediate) vibrational state. ${ }^{18}$ Only before or after the delocalization, rearrangement of solvent/ intermediates can occur (Franck-Condon approximation).

Theoretically, $\Delta G_{\mathrm{TS}}^{\ddagger}$ is composed of entropic $\left(\Delta S_{\mathrm{TS}}^{\ddagger}\right)$ and enthalpic $\left(\Delta H_{\mathrm{TS}}^{\ddagger}\right)$ terms. We found offsets of $\Delta\left(\Delta G_{\mathrm{r}}\right) \approx$ $100 \mathrm{~kJ} \mathrm{~mol}^{-1}\left(\Delta\left(\Delta G_{\mathrm{TS}}^{\ddagger}\right) \approx 13 \mathrm{~kJ} \mathrm{~mol}^{-1}\right)$ between LFERs (Fig. S8E, S11A and S12A, ESI, $\dagger$ involving HA by $\mathrm{HO}^{\bullet}, \mathrm{H}^{\bullet}$ and $\mathrm{CH}_{3}{ }^{\bullet}$, resp.) for non-polar $\left(\mu_{\pi} 0-1 \mathrm{D}\right)$ and zwitterionic compounds $\left(\mu_{\pi}=10-15 \mathrm{D}\right)$. The difference between non-polar $\left(\mu_{\pi}=0-1 \mathrm{D}\right)$ and polar $\left(\mu_{\pi}=5 \pm 2 \mathrm{D}\right)$ compounds is $\Delta\left(\Delta G_{\mathrm{r}}\right) \approx$ $30 \mathrm{~kJ} \mathrm{~mol}^{-1}\left(\Delta\left(\Delta G_{\mathrm{TS}}\right) \approx 4 \mathrm{~kJ} \mathrm{~mol}^{-1}\right.$, involving $\mathrm{HA}$ by $\left.\mathrm{HO}^{\bullet}\right)$. Solvent $\left(\mathrm{H}_{2} \mathrm{O}\right)$ rearrangement enthalpies depend on the polarity of the molecule and its effective solvation, but values in the same range have been reported ${ }^{68-70}$ (note also that 1 hydrogen bond in $\mathrm{H}_{2} \mathrm{O}$ is $\sim 18 \mathrm{~kJ} \mathrm{~mol}^{-1}$ ).

For similar mechanisms such as sequential HAT, $\Delta S_{\mathrm{TS}}^{\ddagger}$ may be a constant factor, in which case $\Delta G_{\text {TS }}^{\ddagger}$ is only determined by $\Delta H_{\mathrm{TS}}^{\ddagger}{ }^{71}$ Thus, $\mu_{\pi}$ is likely to characterize $\Delta H_{\mathrm{TS}}^{\ddagger}$. We infer that $\sigma$ and $\pi$ could characterize $\Delta S_{\mathrm{TS}}^{\ddagger}$, e.g. by distinguishing between concerted and stepwise mechanisms. Analogously, we might discern between outer- and inner-sphere ET via Marcus Theory $^{72}$ (S2, ESI $\dagger$ ). We may further investigate the components of $\Delta G_{\mathrm{TS}}^{ \pm}$by looking at 'exotic' radicals with lower $\Delta\left(\Delta G_{\mathrm{r}}\right)$ (Fig. 1) e.g. 'nucleophillic' tert-butyl or HS'. This may help extending eqn (8) to addition reactions. ${ }^{73,74}$

\subsection{Outlook}

Empirical prediction methods often use many parameters and ambiguous algorithms, which does not facilitate interpretation and can be error-prone. Therefore, one should not accept beforehand that complex methods are needed. Alternatively, TS and frontier molecular orbital theory are closer to chemical intuition than a purely mathematical fitting process. Indicators, or proxies, for the free energy of activation $\Delta G^{\ddagger}$ are more easily retrievable than e.g. with ' $a b$ initio' methods. The approach presented here reflects textbook knowledge of basic chemistry, implying that the parameters used have a physical basis. Despite the uncertainties involved, mathematical fitting yields surprisingly accurate results and may easily compete with machine learning methods with little computational effort. For the examples and controls shown here, it might even compete with computationally expensive quantum chemical (' $a b$ initio') methods. The method presented here can effectively screen a large number of reactions, for multiple radicals.

\section{Summary}

In this study we used a thermodynamic/quantum-mechanical framework and kinetic data to parametrize the Eyring equation to predict the rate constants of one-electron oxidation reactions. Our method assumes a linear-free-energy relationship between activation and reaction energies. Via a thermodynamic cycle we combined frontier orbital interaction, $\left|E_{\mathrm{SOMO}}-E_{\mathrm{HOMO}}\right|$ and 
charge (proton) transfer to adequately describe hydrogen abstraction reactions. On the basis of Hammett substituent constants, we inferred that all one-electron oxidation reactions entail similar rate limiting steps. Thus, offsets between different reaction pathways were approximately constant, which we attribute to specific transition state effects. These effects can be described by local polarity (dipole moment, $\mu$ ) and atom type orbital delocalizability indices. Our method, parametrized to $\mathrm{OH}^{\bullet}$, successfully predicted rate constants for $\mathrm{H}^{\bullet}$ and $\mathrm{CH}_{3}{ }^{\bullet}$ and it is within expectation that rate constants for $\mathrm{OOH}^{\bullet}$ and $\mathrm{O}_{2}{ }^{\bullet-}$ can also be evaluated. Predicted and experimental values for $k_{\mathrm{r}}$ show appreciable correlations.

\section{Conflicts of interest}

The authors declare that they have no conflicts of interest.

\section{Acknowledgements}

Funding by the Swiss National Science Foundation (SNSF) is gratefully acknowledged (grant no. 175493). This work is part of the research programme TTW financing the Contaminants of Emerging Concern in the Water Cycle (CERCEC) project number 15759, which is financed by the Dutch Research Council (NWO). Discussions with Minju Lee (Glasgow University) and Jingwen Chen (Dalian University) were greatly appreciated.

\section{References}

1 F. D. Coms, The Chemistry of Fuel Cell Membrane Chemical Degradation, Proton Exchange Membrane Fuel Cells 8, Pts 1 and 2, 2008, vol. 16, issue 2, pp. 235-255.

2 T. M. Nolte and A. M. J. Ragas, A review of quantitative structure-property relationships for the fate of ionizable organic chemicals in water matrices and identification of knowledge gaps, Environ. Sci.: Processes Impacts, 2017, 19(3), 221-246.

3 B. Lipinski, Hydroxyl Radical and Its Scavengers in Health and Disease, Oxid. Med. Cell. Longevity, 2011, 809696.

4 T. G. Truscott, Pulse radiolysis and flash photolysis, in Photobiology, ed. E. Riklis, Springer, Boston, MA, 1991.

5 A. J. Hendriks, How To Deal with 100,000 + Substances, Sites, and Species: Overarching Principles in Environmental Risk Assessment, Environ. Sci. Technol., 2013, 47(8), 3546-3547.

6 ECHA, ECHA's REACH 2018 Roadmap, Helsinki, Finland, 2015.

7 C. J. van Leeuwen and J. L. M. Hermens, Properties of Chemicals and Estimation Methodologies, Risk Assessment of Chemicals: An Introduction, Springer, 1995, pp. 261-262.

8 ECHA, Practical guide How to use and report (Q)SARs, 2016.

9 Development, O.f.E.C.-o.a., Guidance document on the validation of (quantitative) structure-activity relationship (QSAR) models. 2017.
10 D. Gadaleta, et al., Applicability Domain for QSAR Models: Where Theory Meets Reality, Int. J. Quant. Struct.-Prop. Relat., 2016, 1(1), 45-63.

11 P. Carrio, et al., Applicability Domain Analysis (ADAN): A Robust Method for Assessing the Reliability of Drug Property Predictions, J. Chem. Inf. Model., 2014, 54(5), 1500-1511.

12 F. Sahigara, et al., Comparison of Different Approaches to Define the Applicability Domain of QSAR Models, Molecules, 2012, 17(5), 4791-4810.

13 H. Eyring, The Activated Complex in Chemical Reactions, J. Chem. Phys., 1935, 3(2), 107-115.

14 K. Matzjasyewski and T. P. Davis, Theory of radical reactions, in Handbook of radcial polymerization, Wiley-Interscience, Hoboken, 2002.

$15 \mathrm{~T}$. Bucko, Ab initio calculations of free-energy reaction barriers, J. Phys.: Condens. Matter, 2008, 20(6), 064211.

16 L. P. Hammett, The effect of structure upon the reactions of organic compounds benzene derivatives, J. Am. Chem. Soc., 1937, 59, 96-103.

17 C. Hansch, A. Leo and R. W. Taft, A Survey of Hammett Substituent Constants and Resonance and Field Parameters, Chem. Rev., 1991, 91(2), 165-195.

18 S. Braun-Sand, M. H. M. Olsson and A. Warshell, Proton transport in carbonic anhydrase as an example of the difference between microscopic and phenomenological LFERs, in Computer modelling of enzyme catalysis and its relationship to concepts in physical organic chemistry, in Advances in Physical Organic Chemistry, ed. J. P. Richard, Elsevier, Amsterdam, 2005, pp. 212-217.

19 E. Rorije, et al., Modeling reductive dehalogenation with quantum chemically derived descriptors, SAR QSAR Environ. Res., 1995, 4(4), 237-252.

20 L. H. Jing, J. J. Nash and H. I. Kenttamaa, Correlation of hydrogen-atom abstraction reaction efficiencies for aryl radicals with their vertical electron affinities and the vertical ionization energies of the hydrogen-atom donors, J. Am. Chem. Soc., 2008, 130(52), 17697-17709.

21 T. M. Nolte and W. J. G. M. Peijnenburg, Use of quantumchemical descriptors to analyse reaction rate constants between organic chemicals and superoxide/hydroperoxyl $\left(\mathrm{O}_{2}^{\bullet-} / \mathrm{HO}_{2}^{\bullet}\right)$, Free Radical Res., 2018, 52(10), 1118-1131.

22 E. Rosta and A. Warshel, On the Origins of the Linear Free Energy Relationships: Exploring the Nature of the OffDiagonal Coupling Elements in $\mathrm{S}(\mathrm{N}) 2$ Reactions, J. Chem. Theory Comput., 2012, 8(10), 3574-3585.

23 R. W. Taft, et al., Linear Solvation Energy Relations, J. Solution Chem., 1985, 14(3), 153-186.

24 S. S. Shaik, The Lego Way - Curve Crossing Diagrams as General-Models in Physical Organic-Chemistry, Pure Appl. Chem., 1991, 63(2), 195-204.

25 G. Klopman, Chemical-reactivity and the concept of chargecontrolled and frontier-controlled reactions, Curr. Cont. Eng. Technol. Appl. Sci., 1984, (20), 16.

26 K. Fukui and H. Fujimoto, Frontier orbitals and reaction paths, World Scientific Publishing, Singapore, 1997. 
27 R. G. Pearson, Hard and Soft Acids and Bases, J. Am. Chem. Soc., 1963, 85(22), 3533-3539.

28 T. L. Ho, Hard and Soft Acids and Bases in Organic Chemistry, Academic Press, New York, 1977.

29 W. B. Jensen, Lewis Acid-Base Definitions - Status-Report, Chem. Rev., 1978, 78(1), 1-22.

30 M. Jonsson, Redox Chemistry and Energetics of Radical Cations od Substituted Benzenes, in Radication Chemistry Present Status and Future Trends, ed. B. S. M. Rao and C. D. Jonah, Elsevier Science B.V., 2001.

31 A. M. D. Nicholas and D. R. Arnold, Thermochemical Parameters for Organic Radicals and Radical Ions 1. The Estimation of the $\mathrm{p} K_{\mathrm{a}}$ of Radical Cations Based on Thermochemical Calculations, Can. J. Chem., 1982, 60(17), 2165-2179.

32 T. Nauser, et al., Why Selenocysteine Replaces Cysteine in Thioredoxin Reductase: A Radical Hypothesis, Biochemistry, 2014, 53(30), 5017-5022.

33 K. Fukui, H. Kato and T. Yonezawa, A New QuantumMechanical Reactivity Index for Saturated Compounds, Bull. Chem. Soc. Jpn., 1961, 34(8), 1111-1115.

34 P. F. Barbara, T. J. Meyer and M. A. Ratner, Contemporary issues in electron transfer research, J. Phys. Chem., 1996, 100(31), 13148-13168.

35 ChemAxon, Calculator Plugin for structure property prediction. MarVin Version 5.2.0.

36 P. Gramatica and E. Papa, Screening and ranking of POPs for global half-life: QSAR approaches for prioritization based on molecular structure, Environ. Sci. Technol., 2007, 41(8), 2833-2839.

37 X. Luo, et al., Development of a QSAR model for predicting aqueous reaction rate constants of organic chemicals with hydroxyl radicals, Environ. Sci.: Processes Impacts, 2017, 19(3), 350-356.

38 P. W. N. M. van Leeuwen, Section 1.5.3 - Linear Free Energy Relationships, in Homogeneous Catalysis: Understanding the Art, 2004.

39 S. Endo and K. Goss, Applications of Polyparameter Linear Free Energy Relationships in Environmental Chemistry, Environ. Sci. Technol., 2014, 48(21), 12477-12491.

40 M. T. Baumgartner, et al., Theoretical study of the relative reactivity of chloroethenes with atmospheric oxidants $(\mathrm{OH}$, NO3, $\mathrm{O}(\mathrm{P}-3), \mathrm{Cl}(\mathrm{P}-2)$ and $\mathrm{Br}(\mathrm{P}-2))$, Phys. Chem. Chem. Phys., 2002, 4(6), 1028-1032.

$41 \mathrm{~J}$. Tauber, D. Imbri and T. Opatz, Radical Addition to Iminium Ions and Cationic Heterocycles, Molecules, 2014, 19(10), 16190-16222.

42 N. R. Greiner, Hydroxyl Radical Kinetics by Kinetic Spectroscopy 6. Reactions with Alkanes in Range 300-500 Degrees K, J. Chem. Phys., 1970, 53(3), 1070-1076.

43 E. S. C. Kwok and R. Atkinson, Estimation of Hydroxyl Radical Reaction-Rate Constants for Gas-Phase OrganicCompounds Using a Structure-Reactivity Relationship - an Update, Atmos. Environ., 1995, 29(14), 1685-1695.

$44 \mathrm{~J}$. Markelj and M. Pompe, Modeling of atmospheric $\mathrm{OH}$ reaction rates using newly developed variable distance weighted zero order connectivity index, Atmos. Environ., 2016, 131, 418-423.
45 G. V. Buxton, C. L. Greenstock, W. P. Helman and A. B. Ross, Critical Review of Rate Constants for Reactions of Hydrated Electrons, Hydrogen Atoms and Hydroxyl Radicals (.OH/.O-) in Aqueous Solution., J. Phys. Chem. Ref. Data, 1988, 17, 513.

46 J. Kostal, Computational Chemistry in Predictive Toxicology: status quo et quo vadis?, Advances in Molecular Toxicology, 2017, vol. 10, pp. 139-186.

47 J. K. Thomas, Pulse Radiolysis of Aqueous Solutions of Methyl Iodide and Methyl Bromide. Reactions of Iodine Atoms and Methyl Radicals in Water, J. Phys. Chem., 1967, 71(6), 1919-1925.

48 Y. N. Wang, et al., Estimation of Aqueous-Phase Reaction Rate Constants of Hydroxyl Radical with Phenols, Alkanes and Alcohols, QSAR Comb. Sci., 2009, 28(11-12), 1309-1316.

49 T. M. Nolte and W. J. G. M. Peijnenburg, Aqueous-phase photooxygenation of enes, amines, sulfides and polycyclic aromatics by singlet $(\mathrm{a} 1 \Delta \mathrm{g})$ oxygen: prediction of rate constants using orbital energies, substituent factors and quantitative structure-property relationships, Environ. Chem., 2018, 14(7), 442-450.

50 X. Xu and X. G. Li, QSAR for Predicting Biodegradation Rates of Polycyclic Aromatic Hydrocarbons in Aqueous Systems, Chin. J. Struct. Chem., 2012, 31(8), 1212-1221.

51 J. Gasteiger, et al., Finding the 3D structure of a molecule in its IR spectrum, Fresenius' J. Anal. Chem., 1997, 359(1), 50-55.

52 J. H. Schuur, P. Selzer and J. Gasteiger, The coding of the three-dimensional structure of molecules by molecular transforms and its application to structure-spectra correlations and studies of biological activity, J. Chem. Inf. Comput. Sci., 1996, 36(2), 334-344.

53 P. M. Morse, Diatomic molecules according to the wave mechanics. II. Vibrational levels, Phys. Rev., 1929, 34(1), 57-64.

54 F. De Vleeschouwer, et al., Electrophilicity and nucleophilicity index for radicals, Org. Lett., 2007, 9(14), 2721-2724.

55 E. N. Sharp, P. Rupper and T. A. Miller, The structure and spectra of organic peroxy radicals, Phys. Chem. Chem. Phys., 2008, 10(27), 3955-3981.

56 N. Santschi and T. Nauser, An Experimental Radical Electrophilicity Index, ChemPhysChem, 2017, 18(21), 2973-2976.

57 A. Bottoni, J. Fossey and D. Lefort, On the Role of the Orbital Interaction Concept in the Interpretation of Organic Free Radical Structures and Reactivities, in Molecules in Physics, Chemistry and Biology, ed. J. Maruani, Kluwer Academic Publishers, Dordrecht, 1989, pp. 173-219.

58 A. Borgoo, M. R. Godefroid and P. Geerlings, Atomic Density Functions: Atomic Physics Calculations Analyzed with Methods from Quantum Chemistry, Advances in the Theory of Quantum Systems in Chemistry and Physics, 2012, vol. 22, pp. 139-171.

59 R. M. V. Abreu, I. C. F. R. Ferreira and M. J. R. P. Queiroz, QSAR model for predicting radical scavenging activity of di(hetero)arylamines derivatives of benzo[b]thiophenes, Eur. J. Med. Chem., 2009, 44(5), 1952-1958.

$60 \mathrm{~J}$. M. Mayer and I. J. Rhile, Thermodynamics and kinetics of proton-coupled electron transfer: stepwise vs. concerted 
pathways, Biochim. Biophys. Acta, Bioenerg., 2004, 1655(1-3), 51-58.

61 F. G. Bordwell, et al., Bond-Dissociation Energies in Dmso Related to the Gas-Phase, J. Am. Chem. Soc., 1991, 113(26), 9790-9795.

62 F. P. Tully, et al., Kinetics of the Reactions of Hydroxyl Radical with Benzene and Toluene, J. Phys. Chem., 1981, 85(15), 2262-2269.

63 M. Pompe, et al., Using variable and fixed topological indices for the prediction of reaction rate constants of volatile unsaturated hydrocarbons with $\mathrm{OH}$ radicals, Molecules, 2004, 9(12), 1160-1176.

64 D. R. Lide, Handbook of Chemistry and Physics, CRC Press, Boca Raton, FL, 80th edn, 1999.

65 N. J. Turro, Theoretical organic photochemistry, section 7.5, Modern Molecular Photochemistry, University Science Books, US, 1991, ch. 7, p. 207.

66 L. Munoz-Rugeles and J. R. Alvarez-Idaboy, A proton-electron sequential transfer mechanism: theoretical evidence about its biological relevance, Phys. Chem. Chem. Phys., 2015, 17(43), 28525-28528.

67 L. Blancafort and A. A. Voityuk, Exciton delocalization, charge transfer, and electronic coupling for singlet excitation energy transfer between stacked nucleobases in DNA: An MS-CASPT2 study, J. Chem. Phys., 2014, 140(9), 095102.
68 D. Sakic, H. Zipse and V. Vrcek, Base-catalyzed reactions of environmentally relevant N-chloro-piperidines. A quantumchemical study, Org. Biomol. Chem., 2011, 9(11), 4336-4346.

69 J. J. C. Teixeira-Dias, et al., Transition states for $\mathrm{H}$ atom transfer reactions in the $\mathrm{CH} 2 \mathrm{CH} 2 \mathrm{OH}$ radical: The effect of a water molecule, Phys. Chem. Chem. Phys., 2003, 5(22), 5063-5069.

70 V. Vaquero, et al., Alanine Water Complexes, J. Phys. Chem. A, 2014, 118(14), 2584-2590.

71 I. H. Um, J. E. Park and Y. H. Shin, Combined dual substituent constant and activation parameter analysis assigns a concerted mechanism to alkaline ethanolysis at phosphorus of Y-substituted phenyl diphenylphosphinates, Org. Biomol. Chem., 2007, 5(21), 3539-3543.

72 R. G. Endres, Inner-sphere reorganization energies of outersphere self-exchange reactions utilizing density functional theory, in Trends in Chemical Physics Research, ed. A. N. Linke, Nova Science Publishers, Inc., New York, 2006, ch. 7, p. 140.

73 C. J. Chen and J. W. Bozzelli, Analysis of tertiary butyl radical plus $\mathrm{O}-2$, isobutene plus $\mathrm{HO} 2$, isobutene plus $\mathrm{OH}$, and isobutene-OH adducts plus O-2: A detailed tertiary butyl oxidation mechanism, J. Phys. Chem. A, 1999, 103(48), 9731-9769.

74 N. J. Khalil, Factors Controlling the Addition of Tert-Butyl Radical to 1,2-disubstituted and 1,1,2-trisubstituted Alkenes, Tikrit J. Pure Sci., 2011, 16(2), 75-79. 